(RESEARCH ARTICLE)

\title{
Hard tissue inducibility of a novel synthetic peptide in rat pulp
}

\author{
Nozomi Matsuo ${ }^{1}$, Norimasa Tsuji ${ }^{2}$, Kazuya Tominaga ${ }^{3}$ and Hiroshi Maeda ${ }^{2, *}$ \\ ${ }^{1}$ Graduate School of Dentistry (Endodontics), Osaka Dental University, 8-1 Kuzuhahanazono-cho, Hirakata-shi, Osaka \\ 573-1121, Japan. \\ 2 Department of Endodontics, Faculty of Dentistry, Osaka Dental University, 8-1 Kuzuhahanazono-cho, Hirakata-shi, \\ Osaka 573-1121, Japan. \\ ${ }^{3}$ Department of Oral pathology, Faculty of Dentistry, Osaka Dental University, 8-1 Kuzuhahanazono-cho, Hirakata-shi, \\ Osaka 573-1121, Japan.
}

Publication history: Received on 17 December 2020; revised on 24 December 2020; accepted on 26 December 2020

Article DOI: https://doi.org/10.30574/wjarr.2020.8.3.0495

\begin{abstract}
Enamel matrix derivative (EMD) is a periodontal tissue regeneration material that induced hard tissue formation. This study was performed to examine the reaction of dental pulp tissue directly capped with a synthetic peptide prepared based on EMD research, and to investigate its potential as a pulp capping material.

The experiments were performed in the maxillary left and right first molars of five 6-week-old male Sprague-Dawley rats. The exposed pulp was capped with synthetic peptide in the peptide group, but left uncapped in the control group. Two weeks later, the maxillary bone was isolated and photographed by microfocus computed tomography (CT). The opaque area and volume of the coronal pulp cavity were measured on CT images, and the area-to-volume ratio was calculated. After decalcification, tissue samples were prepared, stained with hematoxylin and eosin (H\&E), and examined histopathologically.

CT images for both groups showed an opaque area in the coronal pulp cavity, and the fraction of images showing opacity was significantly higher in the peptide group $(\mathrm{P}<0.01)$. A large amount of hard tissue was formed in the coronal pulp cavity in the peptide group, around which aggregation of cells with eosinophilic substances in polytopes was observed. Only slight hard tissue formation was observed in the control group. As it showed eosinophilicity on H\&E staining, the synthetic peptide was suggested to be involved in hard tissue formation. Synthetic peptides can potentially be used as pulp capping materials.
\end{abstract}

Keywords: Direct pulp capping; Rat molars; Enamel matrix derivative; Hard tissue formation; A Synthetic peptide

\section{Introduction}

Periodontal tissue regeneration therapy is currently performed using enamel matrix derivative (EMD), which is widely recognized to have regeneration potential [1 - 5]. However, problems associated with uncontrolled pathogens cannot be completely eliminated, because EMD is a biological material extracted from the tooth germ of young pigs [6]. This in fact results in refusal by some patients.

Kim et al. confirmed that eosinophilic round bodies and cartilage-like tissue were formed when EMD was injected subcutaneously into the backs of rats [7]. They also found that the fragment obtained by Matrix-Assisted Laser Desorption/Ionization-Time Of Flight (MALDI-TOF) mass spectrometry (MS) of the round bodies contained a common

\footnotetext{
${ }^{*}$ Corresponding author: Hiroshi Maeda

Osaka Dental University, Department of Endodontics 8-1 Kuzuhahanazonocho Hirakata, Osaka 573-1121, JAPAN.
} 
sequence of seven amino acids, WYQNMIR, which was confirmed to be a partial sequence of exon 5 of porcine amelogenin. Cattle also have the same sequence.

Hida et al. inoculated a peptide newly synthesized from the above sequence (hereinafter referred to as synthetic peptide) subcutaneously into rats, and confirmed ectopic bone, cartilage, and endochondral ossification 2 weeks later, suggesting that synthetic peptides may have potential to induce hard tissue differentiation [8].

In endodontic therapy, on the other hand, it was reported that EMD promoted the differentiation of human dental pulp cells into hard tissue-forming cells resulting in hard tissue formation [9]. It has also been reported that odontoblasts were found in the capped area when an exposed area of dental pulp was directly capped with EMD, suggesting that EMD is useful as a pulp capping material [10]. It is possible that EMD is also involved in the formation of hard tissue in the pulp.

Kato et al. reported upregulation of proliferation and potential to differentiate into hard tissue-forming cells when dental pulp stem cells were cultured with synthetic peptide, suggesting that it was involved in the formation of hard tissue from dental pulp cells in vitro [11]. However, there have been no studies to date on the application of synthetic peptides to dental pulp tissue in vivo, and its usefulness in the formation of hard tissue in dental pulp has not been investigated.

This study was performed to examine the dental pulp tissue following direct capping of the exposed surface with synthetic peptides in rats, and to explore the possibility of using synthetic peptides as direct pulp capping materials.

\section{Material and methods}

\subsection{Synthetic Peptide}

Synthetic peptide was prepared from the seven-amino acid sequence reported by Kim et al. [7] using the method of Hida et al. [8]. Briefly, $0.5 \mathrm{~mL}$ of ultrapure water was added to $7.5 \mathrm{mg}$ of synthetic peptide as described by Noguchi et al. [12]. After filter sterilization, $7.5 \mathrm{mg}$ of propylene glycol alginate was added (Junsei Chemical Co., Ltd., Tokyo, Japan), and the resultant mixture was used in the form of a gel.

\subsection{Direct Pulp Capping}

This experiment was carried out with the approval of the Animal Experiment Committee of Osaka Dental University (approval number: No. 20-040008).

Five 6-week-old male Sprague-Dawley rats (Shimizu Laboratory Supplies Co., Ltd., Kyoto, Japan) were used in our experiment.

After induction of general anesthesia by intraperitoneal administration of a cocktail of $0.75 \mathrm{mg} / \mathrm{kg}$ of medetomidine (Domitor®; Zenoaq, Koriyama, Japan), $2.0 \mathrm{mg} / \mathrm{kg}$ of midazolam (Sand®; Sandoz, Ueyama, Japan), and $2.5 \mathrm{mg} / \mathrm{kg}$ of butorphanol (Betrfanol ®; Meiji Seika Pharma Co., Ltd., Tokyo, Japan), we ensured protection from moisture using a rubber dam sheet (Morita, Tokyo, Japan) cut into a $3 \mathrm{~cm} \times 3 \mathrm{~cm}$ square and a clamp (YDM, Tokyo, Japan).

Direct pulp capping was performed with reference to Dianat et al. [13] and Trongkij et al. [14]. A \# 1/2 round bur (Morita, Tokyo, Japan) was used to form a cavity in the occlusal mesial surface on the maxillary first molar, and dental pulp was exposed with an explorer (YDM). The exposed pulp surface was then washed with physiological saline. After confirming hemostasis, the peptide group (5 teeth in 5 animals) was prepared by capping with the synthetic peptide using a microbrush (Microbrush, Denver, IL, USA). The corresponding contralateral teeth in which the exposed pulp surface was not capped with synthetic peptide were used as controls (5 teeth in 5 animals) after washing with physiological saline and confirming hemostasis. After bonding (SELF CONDITIONER; GC Corp., Tokyo, Japan), the tooth cavity was restored using photopolymerizable resin-reinforced glass ionomer cement (Fujifil LC Flowß; GC Corp.).

\subsection{Sample Extraction and Fixation}

Fourteen days after the treatments, animals were euthanized by an overdose of intraperitoneal pentobarbital (Somnopentyl; Kyoritsu Seiyaku, Tokyo, Japan), and fixed by perfusion with $10 \%$ neutral buffered formalin. Then, the maxilla was removed and fixed by immersion with the same fixative. 


\subsection{Microfocus CT Imaging}

The extracted and fixed maxillae were divided into left and right sides, and the parts including the maxillary first molars were placed into tubes containing 10\% neutral buffered formalin and imaged by microfocus CT (SkyScan1275; Bruker, Billerica, MA, USA) with a tube voltage of $65 \mathrm{kV}$ and current of $85 \mu \mathrm{A}$. The opaque area generated by the coronal pulp cavity and its volume were measured on the CT images. From the obtained results, the proportion of opaque area in the coronal pulp cavity was calculated (Table 1). Image analysis was performed using CTAN software (Bruker).

\subsection{Statistical Analysis}

We set a significance level of $1 \%$ for the $t$ test in examination of the proportion of opacity on the coronal pulp cavity on CT images.

\subsection{Preparation of Tissue Samples and Staining}

EDTA-2Na dihydrate (Hayashi Junyaku Kogyo, Osaka, Japan), tris-aminomethane (Hayashi Junyaku Kogyo), and ultrapure water were mixed to prepare a 10\% EDTA solution (pH 7.4). After decalcification of extracted samples for 4 weeks, they were embedded in paraffin. Then, tissue samples of sagittal slices $6 \mu \mathrm{m}$ thick were prepared. Samples were then stained with hematoxylin eosin (H\&E) according to the conventional method, and characterized by histopathological examination.

\section{Results}

\subsection{Microfocus CT Imaging}

In the peptide group, areas of opacity were found in the coronal pulp cavity in all teeth. The opacity spread widely over the entire coronal pulp cavity, but not on the exposed pulp surface. No opacity was found at the roots of the teeth (Figs. 1 and 2)

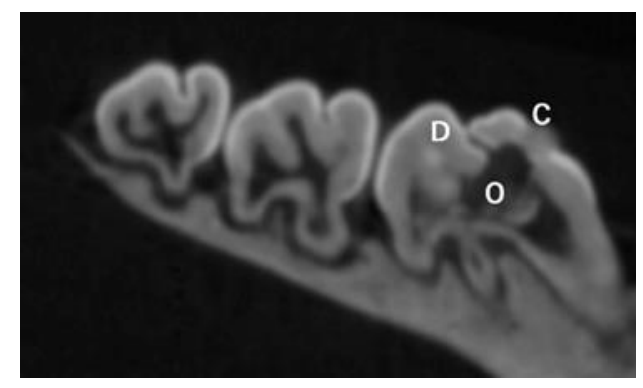

Figure 1 Microfocus CT images of the peptide group. Opacity was seen in the central part of the coronal pulp cavity. C: Cavity, D: Dentin, O: Opacity

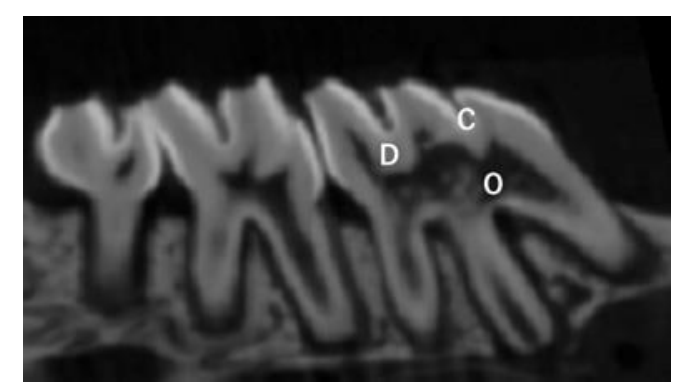

Figure 2 Microfocus CT images of the peptide group. Opacity was widely seen throughout the coronal pulp cavity from the central to the distal part.

C: Cavity, D: Dentin, O: Opacity

In the control group, however, only a small area of opacity was found in the coronal pulp cavity in all teeth. The opacity was only observed in the floor of the pulp chamber. In addition, no opacity was observed at the roots of the teeth (Figs. 3 and 4) 


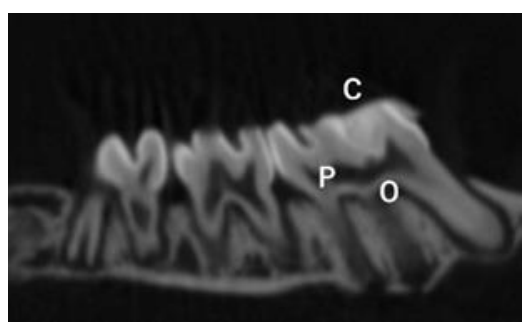

Figure 3 Microfocus CT images of the control group. Slight opacity was seen at the floor of the pulp chamber. C: Cavity, D: Dentin, O: Opacity

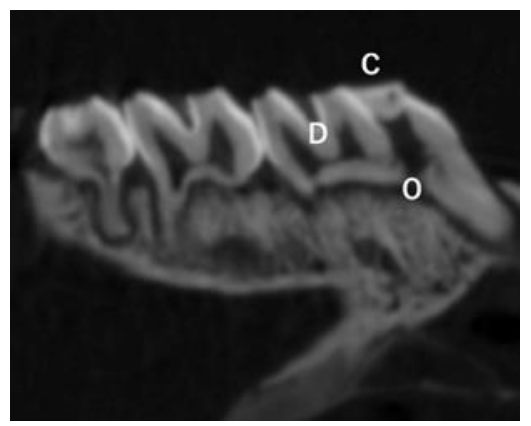

Figure 4 Microfocus CT images of the control group. Slight opacity was seen at the floor of the pulp chamber.

C: Cavity, D: Dentin, O: Opacity

Table 1 shows the volume and fraction of opacity in the pulp cavity in the peptide and control groups. The average volume of opacity and that of the pulp cavity were $2.34 \times 10-3 \pm 3.40 \times 10-3 \mathrm{~mm} 3$ and $0.25 \pm 1.86 \times 10-2 \mathrm{~mm} 3$, respectively, in the peptide group. The average fraction of opacity in the pulp cavity was $9.52 \% \pm 1.68 \%$. On the other hand, the average volumes of opacity and of the pulp cavity were $6.45 \times 10-3 \pm 4.57 \times 10-3 \mathrm{~mm} 3$ and $0.24 \pm 1.14 \times$ $10-2 \mathrm{~mm} 3$, respectively, in the control group. The average fraction of opacity in the pulp cavity in this group was $2.68 \%$ $\pm 0.13 \%$

Table 1 Volume and fractions of opacity in the coronal pulp cavities of the peptide and control groups

\begin{tabular}{|c|c|c|c|c|c|c|}
\hline \multirow[b]{2}{*}{$\begin{array}{l}\text { Sr. } \\
\text { No. }\end{array}$} & \multicolumn{3}{|l|}{ Peptide group } & \multicolumn{3}{|l|}{ Control group } \\
\hline & Opacity $\left(\mathrm{mm}^{3}\right)$ & $\begin{array}{l}\text { Coronal Pulp cavity } \\
\left(\mathbf{m m}^{3}\right)\end{array}$ & $(\%)$ & Opacity $\left(\mathrm{mm}^{3}\right)$ & $\begin{array}{l}\text { Coronal Pulp cavity } \\
\left(\mathbf{m m}^{3}\right)\end{array}$ & (\%) \\
\hline 1 & 0.02674 & 0.27240 & 9.82 & 0.00603 & 0.23473 & 2.57 \\
\hline 2 & 0.01999 & 0.26533 & 7.53 & 0.00630 & 0.24250 & 2.60 \\
\hline 3 & 0.02185 & 0.24822 & 8.80 & 0.00722 & 0.25326 & 2.85 \\
\hline 4 & 0.02781 & 0.22972 & 12.11 & 0.00624 & 0.22368 & 2.79 \\
\hline 5 & 0.02197 & 0.23490 & 9.35 & 0.00644 & 0.24627 & 2.62 \\
\hline
\end{tabular}




\subsection{Statistical Analyses}

Student's $t$ test indicated that the fraction of opacity in the dental pulp cavity was significantly higher in the peptide group than the control group ( $\mathrm{P}<0.01$, Fig. 5).

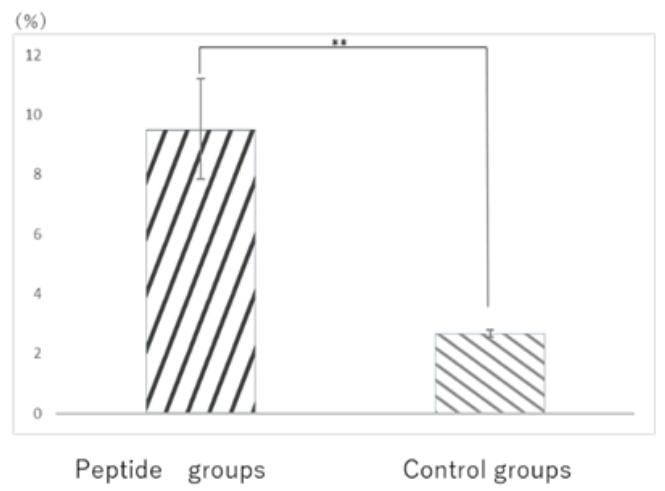

Figure 5 Fractions of opacity in the coronal pulp cavities of the peptide and control groups

\subsection{Tissue Samples}

In the peptide group, hard tissue was observed in the coronal pulp cavity in all teeth, and large areas of hard tissue were formed from the dentin wall of the coronal pulp cavity to the central and distal parts. A large fraction of normal pulp was observed in the distal part, and no hard tissue was found in the root. In addition, aggregation of cells with eosinophilic substances in polytopes was observed near the sites of hard tissue formation. Infiltration of inflammatory cells, such as neutrophils, was observed on the surface of exposed pulp (Figs. 6-9).

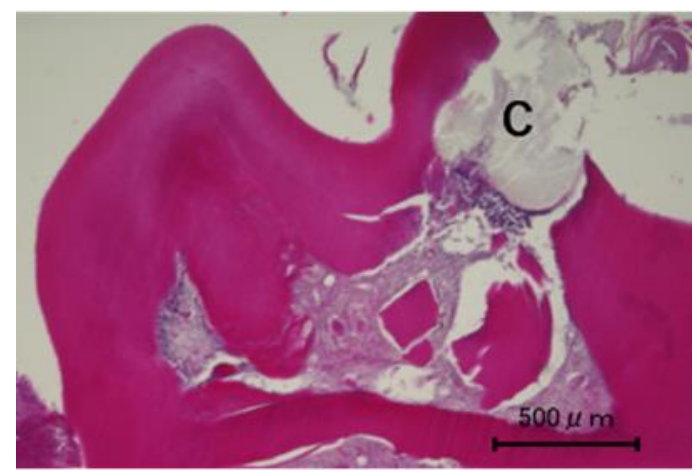

Figure 6 Tissue samples $(4 \times$ ) for the peptide group after H\&E staining. Hard tissue was seen at the distal part of the coronal pulp cavity

\section{C: Cavity}

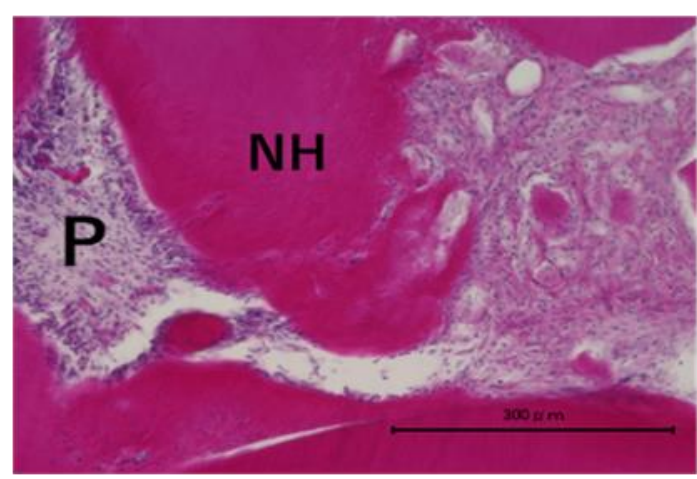


Figure 7 High-magnification view of the centrifugal part of Figure 5 (20x). Aggregation of cells with eosinophilic substances in polytopes was observed near the site of hard tissue formation.

P: Pulp, NH: Newly formed hard tissue

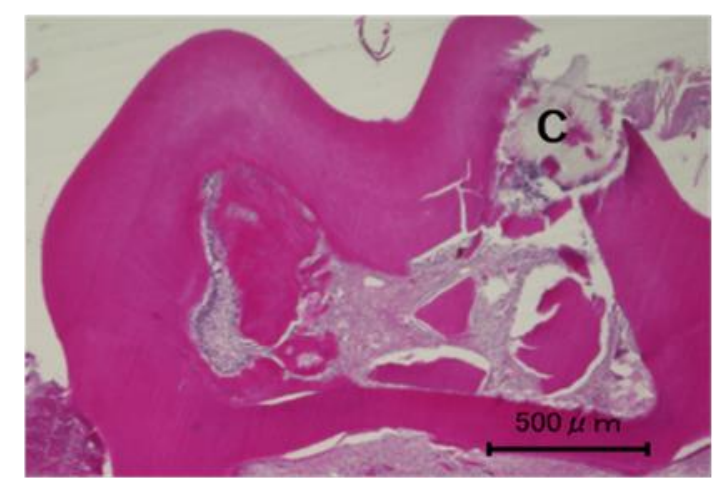

Figure 8 Tissue sample of the peptide group after H\&E staining ( $4 \times$ ). Hard tissue formation was seen at the central part of the coronal pulp

\section{C: Cavity}

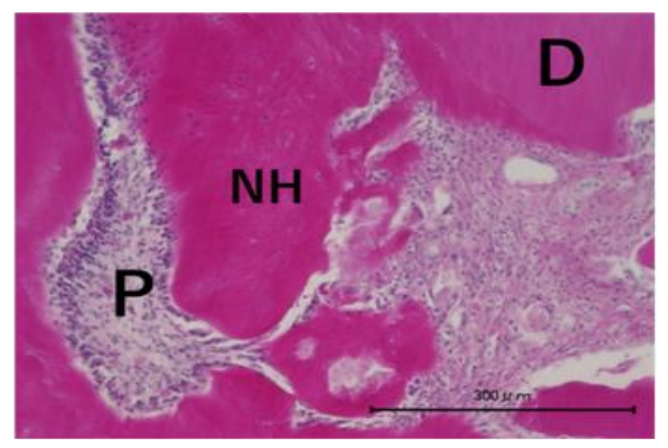

Figure 9 High-magnification view of the central part of Figure 8 (20x). Aggregation of cells with eosinophilic substances in polytopes was observed near the site of hard tissue formation.

\section{D: Dentin, P: Pulp, NH: Newly formed hard tissue}

In the control group, we observed the formation of hard tissue that was connected to the dentin wall of the coronal pulp cavity just below the exposed pulp surface of all teeth. However, only slight hard tissue formation was observed on the dentin wall in the central part of the coronal pulp cavity. In addition, normal pulp was observed in the distal part, and no hard tissue was found in the root. Infiltration of inflammatory cells occurred on the surface of exposed pulp (Figs. 10 -13)

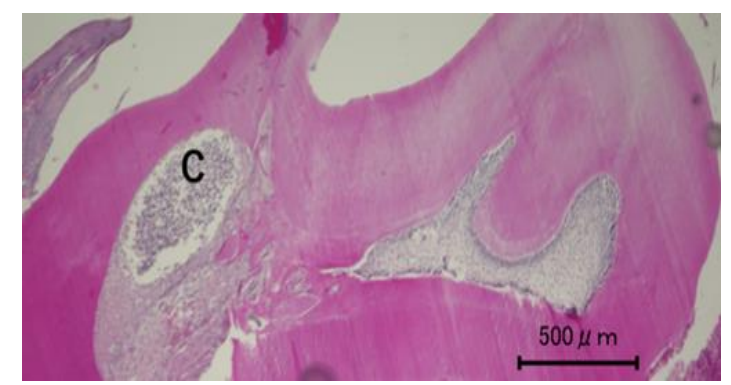

Figure 10 Tissue sample of control group after H\&E staining (4×).

C: Cavity 


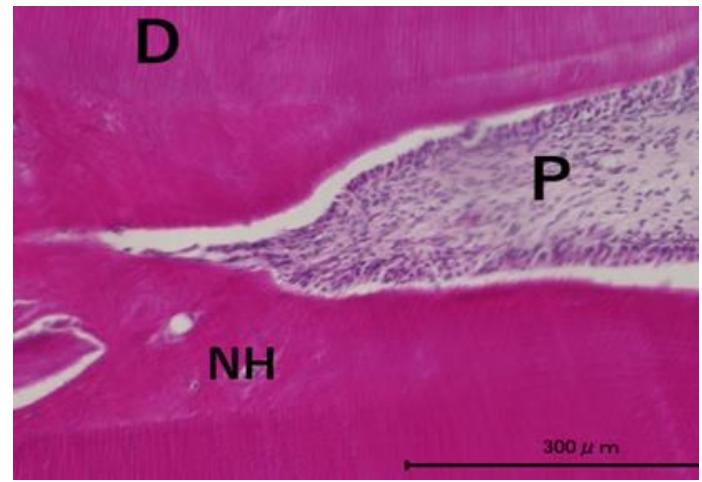

Figure 11 High-magnification view of the central part of Figure $10(20 \times)$. Hard tissue was formed on the floor of the pulp chamber

D: Dentin, P: Pulp, NH: Newly formed hard tissue

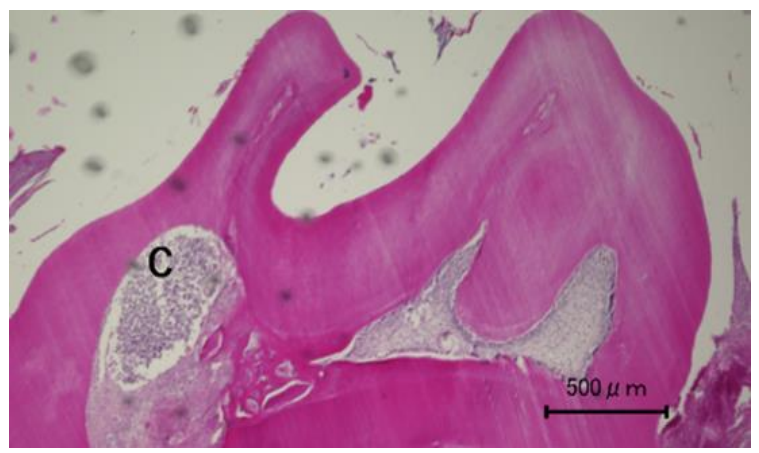

Figure 12 Tissue sample of control group after H\&E staining $(4 \times)$.

\section{C: Cavity}

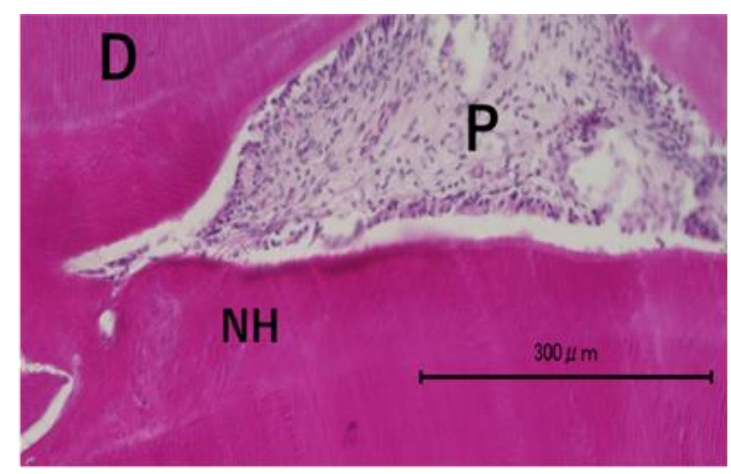

Figure 13 High-magnification view of the central part of Figure 12 (20x). Hard tissue was formed on the floor of the pulp chamber.

D: Dentin, P: Pulp, NH: Newly formed hard tissue

\section{Discussion}

EMD is used for periodontal tissue regeneration therapy, and its effectiveness is well known, including induction of hard tissue formation. However, EMD is a biological material extracted from the tooth germs of young pigs, which leads to refusal in some patients. The EMD-derived synthetic peptide used in the present study can eliminate the abovementioned problems. In addition, it has great potential for development from the viewpoint of its ability to induce hard tissue. 
In endodontic treatment, the possibility of using EMD as a direct pulp capping material is now being investigated, focusing on its ability to induce hard tissue formation. However, even if EMD has potential as a direct pulp capping material, concerns remain about it as a biological material, and there are many issues that must be resolved for clinical application in endodontic treatment. The synthetic peptide used in the present study solved the greatest problem of EMD, and it is expected that our synthetic peptide will be clinically applicable as an alternative to EMD.

In this study, the rat pulp was directly capped with synthetic peptide. The synthetic peptide was prepared as a white powder, which was dissolved in ultrapure water, mixed with propylene glycol alginate, and used in gel form. The concentration of the synthetic peptide was determined with reference to bone defect regeneration experiments in the rat periodontium reported by Noguchi et al. [12]. The optimal concentration of synthetic peptide, however, may differ between pulp tissue and the periodontium with regard to the formation of hard tissue. Further investigations regarding this point are required.

Hard tissue regeneration ability of the synthetic peptide was evaluated by measuring the volumes of the pulp cavity and opacity on microfocus CT and calculating their ratio with reference to Okamoto et al. [15] and Imura et al. [16]. As microfocus CT was performed after removing the maxilla, it was not possible to obtain images of experimental teeth before surgery. Although the amount of regenerated tissue could not be estimated accurately from opacity in the pulp cavity, the imaged indicated the presence of regenerated tissue to some extent.

By analysis of microfocus CT images, the ratio of the volume of opacity to the pulp cavity was shown to be significantly higher in the peptide group than the control group, suggesting regeneration of hard tissue. The regenerated opaque hard tissue was observed widely on the coronal pulp, but not on the exposed surface. This was the first study to use synthetic peptide for dental pulp tissue in vivo, confirming the formation of hard tissue in dental pulp tissue. This result is significant because clinical applicability of synthetic peptide was strongly suggested.

Tissue samples were prepared and analyzed to investigate the dental pulp tissue and regenerated hard tissue after direct pulp capping with synthetic peptide. Infiltration of inflammatory cells was observed on the exposed pulp surface directly capped with synthetic peptide. Inflammation is not desirable. However, this may have been due to the traumatic effects of direct pulp capping, i.e., operational problems during demyelination with an explorer or peptide application. Large amounts of new hard tissue was observed after direct pulp capping with synthetic peptide not on the exposed pulp surface but in the dental pulp tissue just below the exposed pulp surface. Dentin bridges were not formed. This may have been due to inflammation on the exposed surface. In the control group, however, a small amount of newly generated hard tissue was found in the pulp cavity, as observed on microfocus CT images. This is characteristic of rats, in which hard tissue was reported to be formed 10 - 14 days after pulp treatment without the action of drugs [17 - 19]. In addition, aggregation of cells with eosinophilic substances in polytopes was observed near the sites of tissue regeneration in the peptide group. It is likely that the synthetic peptide was involved in the formation of hard tissue, because H\&E staining indicated that the synthetic peptide had eosinophilicity. Ideally, however, the formation of a dentin bridge is desirable in direct pulp capping. It is possible that the dentin bridge did not form due to the properties of the substrate to which the synthetic peptide was applied, and/or the action of the peptide deep in the pulp due to the concentration used in our experiments. In addition, considering the difficulty of operation in small animals, such as rats used in this study, further studies to confirm the pulp reaction in large animals, such as dogs, are required.

\section{Conclusion}

The results of this study suggested that the synthetic peptide used here was involved in hard tissue formation by direct application in pulp capping in rats, and it may be applicable as a pulp capping material. Various issues remain to be investigated in future studies.

\section{Compliance with ethical standards}

\section{Acknowledgments}

This study was conducted at the morphological research facility and laboratory animal facility of the Osaka Dental University Dental Research Institute. This study was supported in part by 2018-2020 (C: 18K09590) Grants-in-Aid for Scientific Research (C) from the Japan Society for the Promotion of Science.

\section{Disclosure of conflict of interest}

All authors declare no conflicts of interest associated with this manuscript. 


\section{Statement of ethical approval}

All procedures in this study were approved by the Animal Experiment Committee of Osaka Dental University and conformed to the guidelines described in the Guiding Principles for the Use of Laboratory Animals (approval no. 20040008).

\section{References}

[1] Ishii T, Miyamoto Y. Periodontal regeneration with EMD (enamel matrix derivative). J Jpn Acad Clin Perio-dontol. 2007; 25: 64 - 69. (Japanese)

[2] Zetterström O, Andersson C, Eriksson L, Fredriksson A, Friskopp J, Heden G, Jansson B, Lundgren T, Nilveus R, Olsson A, Renvert S, Salonen L, Sjöström L, Winell A, Östgren A, Gestrelius S. Clinical safety of enamel matrix derivative (Emdogain $囚$ ) in the treatment of periodontal defects. J Clin Periodontol. 1997; 24: 697 - 704.

[3] Meyle J, Gonzales J.R, Bödeker R.H, Hoffmann T, Rich-ter S, Heinz B, Arjomand M, Reich E, Sculean A, Jepsen K, Jepsen S. A randomized clinical multicentre trial comparing enamel matrix derivative and membrane treatment of buccal class II furcation involvement in mandibular molars. Part III: patient factors and treatment outcome. J Periodontol. 2004; 75: 1188 - 1195.

[4] Rasperini G, Silvestri M, Ricci G. Long-term clinical observation of treatment of infrabony defects with enamel matrix derivative (Emdogain): surgical reentry. Int J Periodontics Restorative Dent. 2005; 25: 121 - 127.

[5] Esposito M, Grusovin MG, Coulthard P, Worthington HV. Enamel matrix derivative (Emdogain) for periodontal tissue regeneration in intrabony defects. A Cochrane systematic review. Int J Oral Implantol (Berl). 2009;2(4):247-266.

[6] Hammarström L, Heiji L, Gestrelius S. Periodontal regeneration in a buccal dehiscence model in monkeys after application of enamel matrix proteins. J Clin Periodontol. 1997; 24: 669 - 677.

[7] Kim N-H, Tominaga K, Tanaka A. Analysis of eosinophilic round bodies formed after injection of enamel matrix derivative into the backs of rats. J Periodontol. 2005; 76: 1934 - 1941.

[8] Hida T, Tominaga K, Tanaka A. Tissue reaction to synthetic oligopeptide derived from enamel matrix derivative in rats. Int J Oral Sci. 2010; 7: 26 - 33.

[9] Riksen EA, Landin MA, Reppe S, Nakamura Y, Lyngstadaas SP, Reseland JE. Enamel matrix derivative promote primary human pulp cell differentiation and mineralization. Int J Mol Sci. 2014; 15: 7731 - 7749.

[10] Orhan EO, Maden M, Senguüven B. Odontoblast-like cell numbers and reparative dentine thickness after direct pulp capping with platelet-rich plasma and enamel matrix derivative: a histomorphometric evaluation. 2012 ; 45 : $317-325$.

[11] Kato H, Taguchi Y, Yamawki I, Ruan Y, Wu Q, Nakano Y, Tsumori N, Nakata T, Noguchi M, Umeda M. Amelogenin exon 5 peptide promotes cell proliferation and osteogenic differentiation in human dental pulp stem cells. Appl Sci. 2019; 9: 4425.

[12] Noguchi M, Tominaga K, Tanaka A, Ueda M. Hard tissue formation induced by synthetic oligopeptide derived from enamel matrix derivative. Oral Med Pathol. 2012; 16: 75 - 80.

[13] Dianat O, Mashhadiabbas F, Ahangari Z, Saedi S, Motamedian SR. Histologic comparison of direct pulp capping of rat molars with MTA and different concentrations of simvastatin gel. J Oral Sci. 2018; 60(1): 57 - 63.

[14] Trongkiji P, Sutimuntanakul S, Lapthanasupkul P, Chaimanakarn C, Wong RH, Banomyong D. Pulpal responses after direct pulp capping with two calcium-silicate cements in a rat model. Dent Mater J. 2019; 38(4): 584 - 590.

[15] Okamoto M, Takahashi Y, Komichi S, Ali M, Yoneda N, Ishimoto T, Nakano T, Hayashi M. Novel evaluation method of dentin repair by direct pulp capping using high-resolution micro-computed tomography. Clin Oral Investig. 2018; 22(8): $2879-2887$.

[16] Imura K, Hasimoro Y, Okada M, Yoshikawa K, Yamamoto K. Application of hydroxyapatite nanoparticleassembled powder using basic fibroblast growth factor as a pulp-capping agent. Dent Mater J. 2019; 38(5): 713 -720 .

[17] Kakehashi S, Stanley H R, Fitzgerald R J. The effects of surgical exposures of dental pulps in germ-free and conventional laboratory rats. Oral Surg. 1965; 20: 340 - 349. 
World Journal of Advanced Research and Reviews, 2020, 08(03), 392-401

[18] Yoshida H. Pathological changes in exposed pulp tissues in germ-free rats. J Stomatol Soc Jpn. 1982; 49: 591 602.

[19] Aoki T. Response of exposed pulp to capping agents in germ-free rat molars. J Stomatol Soc Jpn. 1983; 50(4): 636 -650 . 